\title{
Single shot nanochannel processing with femtosecond Bessel beams
}

\author{
F. Courvoisier (*), M. K. Bhuyan, M. Jacquot, P.-A. Lacourt, \\ R. Salut, L. Furfaro and J. M. Dudley \\ FEMTO-ST Institute, Department of Optics P.M. Duffieux, UMR CNRS 6174, Université de Franche-Comté, \\ 25030 Besançon, France \\ "francois.courvoisier@femto-st.fr
}

Ultrashort laser material processing is a versatile technology that allows material structuring at nanometer scales [1]. In the context of the fabrication of nanofluidics components or photonics crystals, high aspect ratio nanochannel in dielectrics is required. Deep nano-craters have been produced with single shot from gaussian beams by taking advantage of geometrical aberrations and nonlinear propagation to elongate the beam focus [2]. However, the distortion of gaussian beams during nonlinear propagation in dielectrics produces irregular structures with strongly interdependant channel diameter and length.

In this framework, Bessel beams present a significant benefit to maintain intense laser-matter interaction over enhanced propagation lengths since their nonlinear propagation can be stationary [3]. This property has been used to process taper-free micro-channels in the multishot regime [4].

Here, we report the processing of high aspect ratio nano-channels in glass with femtosecond Bessel pulses in the single shot regime. Channels aspect ratio can exceed 100 and diameters range between 200 and $800 \mathrm{~nm}$. Fig. 1(a) shows the focusing geometry of the femtosecond Bessel beam that is produced by a spatial light modulator, and crosses the exit side of the sample. Fig. 1(b-c) show respectively the image of a nanochannel produced with a single shot of energy $0.85 \mu \mathrm{J}$ and an array of nanochannels, showing the repeatability and capability of the technique for the processing of periodic nanostructures [5].
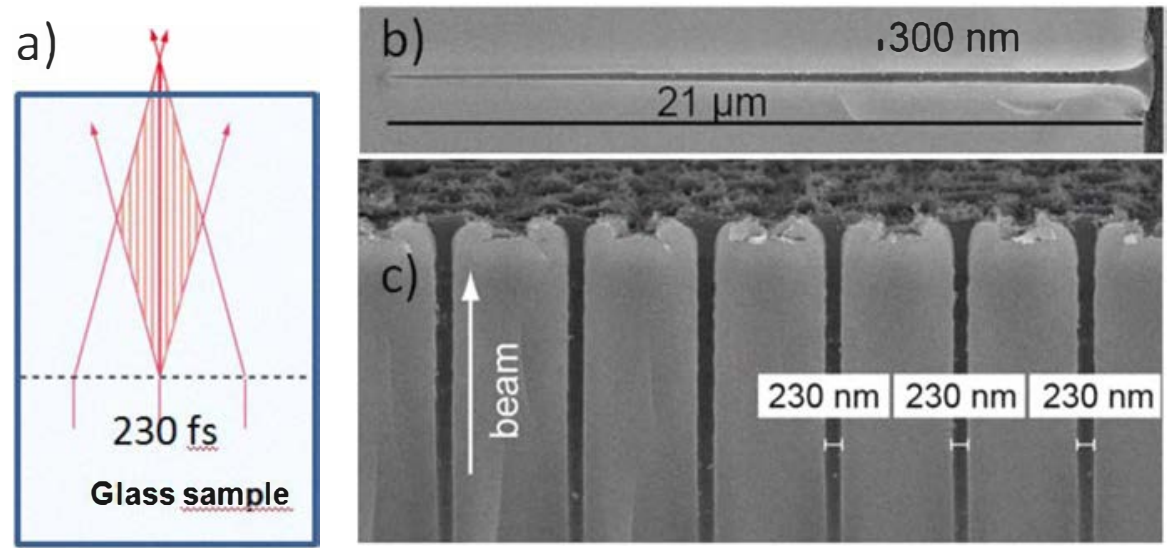

Fig. 1 (a) Focusing geometry of the femtosecond Bessel beam at the exit side of the sample. (b) SEM image of a nanochannel formed by a Bessel pulse of $0.85 \mu \mathrm{J}$. c) SEM image of an array of nanochannels with $1.6 \mu \mathrm{m}$ pitch, processed at $0.7 \mu \mathrm{J} /$ pulse.

One of the main novelties is that nanochannels can be produced with a linear and independent control of channel length and diameter. Through-channels are also demonstrated with single shot illumination.

The stationarity of the nonlinear propagation of Bessel beams at ablation level intensity is key for uniform energy deposition and high aspect ratio nanochannel processing. Underlying physical process and physical limits of the technique will be discussed. Our results represent an important progress of femtosecond laser material nanoprocessing which is applicable to all dielectrics. We anticipate wide application to nanofluidics and nanophotonics.

\section{References}

[1] R. R. Gattass and E. Mazur, "Femtosecond laser micromachining in transparent materials," Nat. Photonics 2, 219 (2008).

[2] Y. V. White, X. Li, Z. Sikorski, L. M. Davis and W. Hofmeister, "Single-pulse ultrafast-laser machining of high aspect nano-holes at the surface of $\mathrm{SiO}_{2}$, ,Opt. Express 16, 14411 (2008).

[3] M. K. Bhuyan, F. Courvoisier, P.-A. Lacourt, M. Jacquot, L. Furfaro, M. J. Withford, and J. M. Dudley, "High aspect ratio taper-free microchannel fabrication using femtosecond Bessel beams," Opt. Express 18, 566-574 (2010)

[4] M. A. Porras, A. Parola, D. Faccio, A. Dubietis and P. Di Trapani, "Nonlinear Unbalanced Bessel Beams: Stationary Conical Waves Supported by Nonlinear Losses," Phys. Rev. Lett. 93, 153902 (2004).

[5] M. K. Bhuyan, F. Courvoisier, P.-A. Lacourt, M. Jacquot, R. Salut, L. Furfaro, and J. M. Dudley "High aspect ratio nanochannel machining using single shot femtosecond Bessel beams," Appl. Phys. Lett. 97, 081102 (2010). 